\title{
Analysis and research of the coverage area of the LoRaWAN gateway in various conditions for smart city applications
}

\author{
V.A. Shpenst \\ D.E.Sc., Professor, Saint-Petersburg Mining University, Russia \\ A.V. Terleev \\ Postgraduate student, Saint-Petersburg Mining University, Russia
}

\begin{abstract}
Smart city services nowadays are gaining popularity worldwide. Russia is not an exception. Since early 2000's smart city market is growing steadily and, according to forecasts, will continue to grow. Today, the possible applications of smart city services cover a wide range of sectors - from energy production, distribution and consumption to sustainable mobility and waste management. All the innovative services require millions of monitoring sensors and control devices to be connected between each other and to a management platform. Hence, new types of wireless communication networks that meet the specific requirements of smart city services are needed. LoRaWAN is the most promising M2M (Machine-to-Machine) communication technology among other LPWANs such as NB-IoT and SigFox. Conducted field study of LoRaWAN gateway coverage in different conditions shows the LoRaWAN attenuation coefficient in conditions of city center and outskirt and reveals the factors on which the signal attenuation coefficient depends.
\end{abstract}

Keywords: Smart city, power line, gateway, filter, detection, Energy efficiency, Frequency

\section{INTRODUCTION}

The first mention of the term "Smart city" date back to the early 2000's (Sikora-Fernandez \& Stawasz, 2016). The smart city concept initially described how to use IT infrastructure to create a virtual entity of a city in the information society (Ishida \& Isbister, 2000). Later, the smart city was mainly associated with the strengthening role of intellectual technologies in increasing the efficiency of urban development (Van der Meer \& Van Winden, 2003). Nowadays, the term "Smart city" has transformed to a "smart sustainable city" (SSC), in which information and communication technologies and other tools, on the one hand, are used to improve the effectiveness of the functioning of the city and the provision of urban services and, as a consequence, the overall quality of life, as well as strengthening competitiveness, and on the other hand, satisfy the needs of present and future generations without negatively affecting the economic, social and ecological components of a city (Smart cities and infrastructure, 2019).

There are no unified criteria to assess the level of city's smartness. Therefore, several approaches used in Russian and international practice can be cited. For instance, the National Research Institute for Technology and Communications (NIITS) has developed the "Smart Cities Indicators" rating that is based on data obtained from public sources and considers 26 indicators characterizing the level of development of 7 key areas of the smart city (Indicators of smart cities NIITS, 2017). Another approach is used by specialists of Skolkovo Business School: the smartness index is calculated for 15 biggest Russian cities, such as Moscow, Saint Petersburg, Kazan, Volgograd, Novosibirsk, 
Yekaterinburg, Nizhny Novgorod, Samara, Chelyabinsk, Omsk, Rostov-on-Don, Ufa, Krasnoyarsk, Perm, Voronezh. The calculation method considers 7 spheres of smart city services implementation: transport, healthcare, public administration, media, education, finance, trade (SKOLKOVO Business School Emerging Market Research Institute, 2019).

It is rather difficult to estimate the actual scale of the global market for smart city technologies. Moreover, it is even more complicated to forecast how they will change in the medium and long term. However, some attempts to predict the evolution of the smart city market are still being made. Thus, according to the estimates of the research company Markets \& Markets, the market volume in 2017 amounted to 424.68 billion US dollars, and by 2022 it will already reach 1.2 trillion US dollars (Markets and Markets [Online],2019). Frost \& Sullivan experts give another estimate: according to their forecasts, the smart city technology market will reach $\$ 2.4$ trillion by 2025 . The market growth is also due to the fact that, as it develops, in addition to traditional IT companies and infrastructure giants, new players begin to emerge - small and medium-sized technology firms, engineering and consulting companies. The estimates of the smart city market volume are varying vastly but it is the obvious fact that the number of smart city services will significantly grow in the nearest future.

Smart city services can be divided into groups according to the sphere of its implementation in the city's ecosystem. In Russia, the following 4 groups of services are in the spotlight nowadays:

I. Smart energy. The group covers the whole chain - from efficient and environmentally friendly energy production (both centralized and decentralized), through distribution system with minimum loses up to responsible consumption on the demand side.

II. Smart and sustainable mobility. The main benefits of the smart mobility introduction are reduced congestion of roads, reduction of negative impact on the environment, as well as reduction of energy consumption of vehicles.

III. Smart waste management. This smart city service implies a transparent control of all the waste flows within the city together with best available technics for waste recycling and utilization.

IV. Smart environmental solutions. The package of technological solutions designed to provide better environmental protection for smart cities includes a whole range of components - these are environmental monitoring systems, smart wastewater treatment systems; and renewable energy solutions. Such solutions contribute to improving the quality of the environment (air, soil, water), the transition to a more rational model of energy use and, as a result, improving the health status of citizens and the sanitary situation in the city as a whole.

It is important to understand that all the services require millions of monitoring sensors and actuators which are connected to the single or several management platforms. Today, the number of connected sensors is growing explosively and has already exceeded the number of people with access to the global network (Evans, 2011). The Figure 1 presents the forecast of number of connected devices and sensors until 2025 in comparison with number of people connected to the Internet.

The upcoming smart city revolution requires an infrastructure that can enable the effective interaction of millions of smart devices and sensors. Such interaction has some specific requirements among which:

1. Transfer of small data packages:

2. Energy efficiency;

3. Ability to connect devices in remote areas;

4. High degree of data protection;

5. Interoperability. 


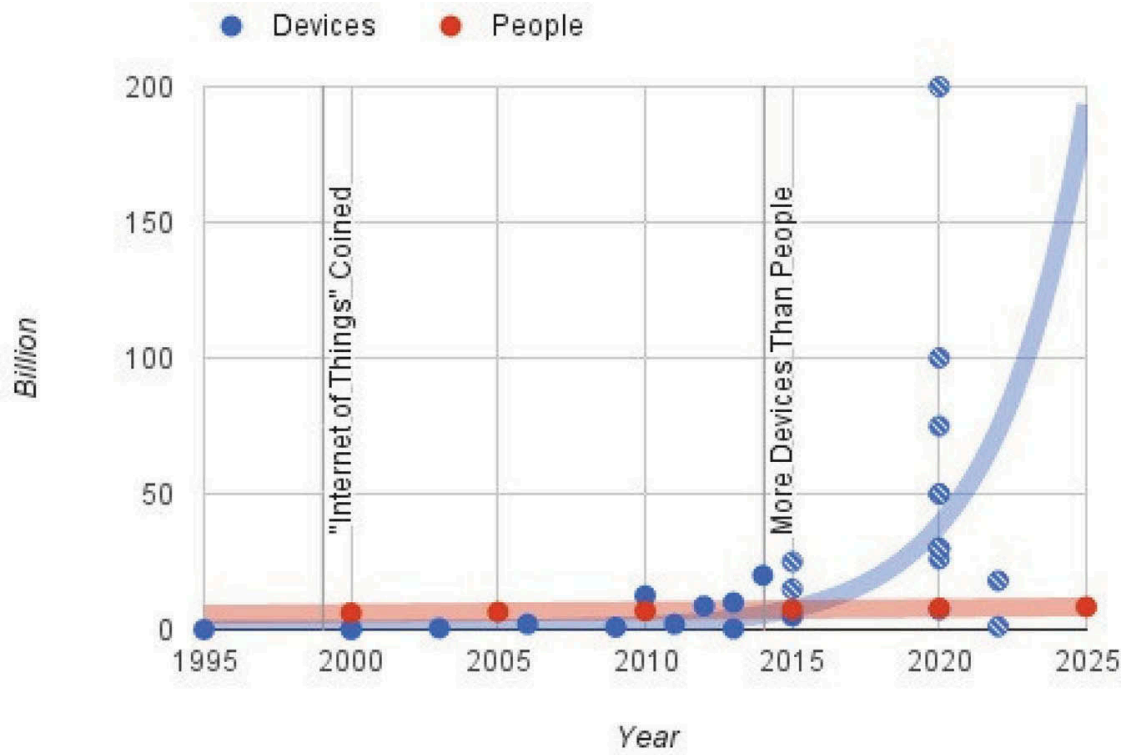

Figure 1. The forecast of number of connected devices and sensors until 2025 (www.brookings.edu).

In practice, energy efficiency is the biggest challenge from the list above. Connected enddevices have to be able to operate for a long period of time (several years) powered by imbedded battery with no connection to the grid. Otherwise the maintenance of the end-devices will be difficult and expensive.

\section{METHODS}

To effectively solve problems related to energy consumption, new types of LPWAN (Low Power Wide Area Networks) have appeared. Technologies that allow connecting autonomous devices to the global network appeared in 2015-2016 and gradually gaining popularity (Koucheryavy \& Vladyko, 2020). The most popular of them are: LoRaWAN, Narrow-band IoT (NB-IoT) and Sigfox. Comparison of the main technical characteristics of the networks is presented in Table 1.

Sigfox is practically not presented in Russia. The first few NB-IoT networks was deployed in Moscow and Saint Petersburg in 2019 by local telecommunication companies. In the same time there are a number of public and private LoRaWAN networks in Russia. It can be explained by affordability of LoRaWAN gateways and end-devices compare to Sigfox and NB-IoT.

Table 1. Comparison of LPWAN.

\begin{tabular}{llll}
\hline Characteristic & LoRa & Sigfox & NB-IoT \\
\hline Modulation method & CSS & - & OFDMA/DSSS \\
Range & ISM & ISM & Licensed \\
Rate & $0.3-50 \mathrm{~Kb} / \mathrm{sec}$ & $100 \mathrm{bit} / \mathrm{sec}$ & $1-200 \mathrm{~Kb} / \mathrm{sec}$ \\
Battery life & up to 10 years & - & up to 10 years \\
Frequency & $868.8 \mathrm{MHz}$ (Europe) & $868.8 \mathrm{MHz}$ (Europe) & $700 / 800 / 900 \mathrm{MHz}$ \\
& $915 \mathrm{MHz}$ (USA) & $915 \mathrm{MHz}$ (USA) & \\
& $433 \mathrm{MHZ}$ (Asia) & & \\
\hline
\end{tabular}




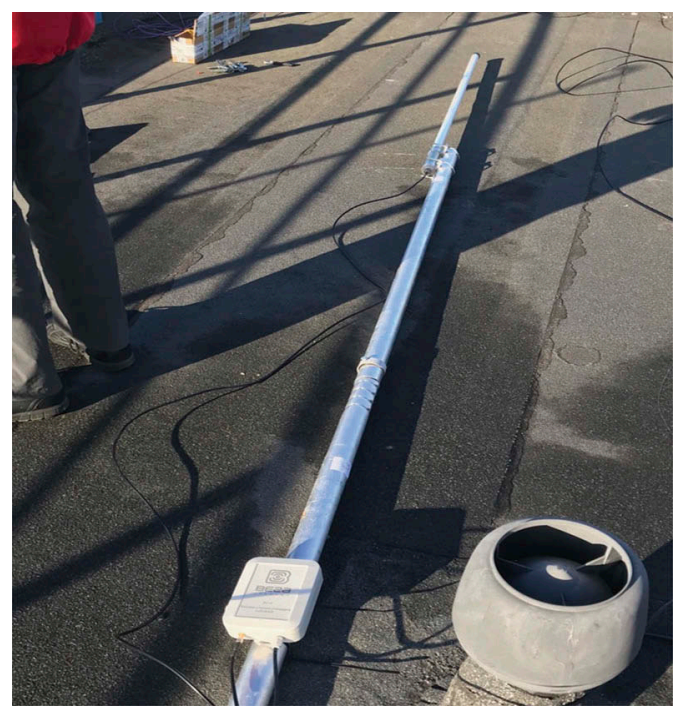

Figure 2. LoRaWAN gateway and antenna.

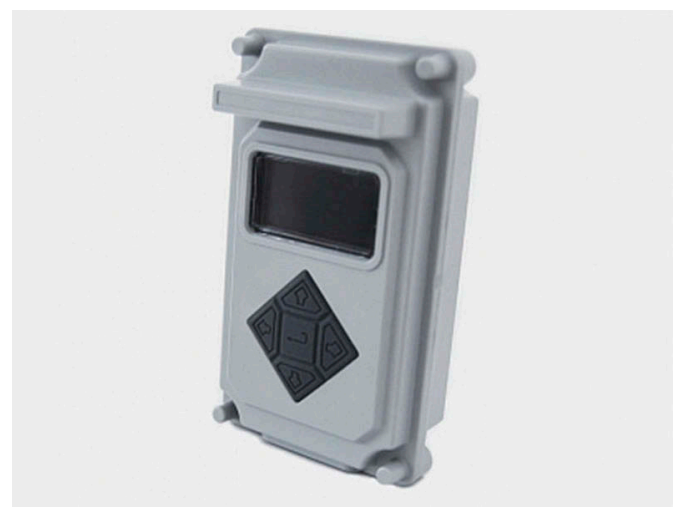

Figure 3. Tester of LoRaWAN network (https://www.euromobile.ru).

In frame of the research the coverage of LoRaWAN gateway in different conditions has been studied. The conditions were (a) city center and (b) outskirt surrounded by coniferous forest. Inboth locations LoRaWAN gateway with vertical omnidirectional antenna (864-876 $\mathrm{MHz} 10 \mathrm{dBi}$ ) were used (Figure 2).

Coverage area was assessed by measuring the special network tester (Figure 3) at preapproved control points. This device sends a special signal to the LoRaWAN network, in response to which the network informs it of the number of gateways that have received this signal and the signal quality. The tester displays this data every time the button is pressed. The device is used to test LoRaWAN networks when they are deployed and configured.

The gateway was stationary at the height of $25 \mathrm{~m}$ above the ground, and the tester moved a predetermined distance from the gateway in the range from 50 to $1500 \mathrm{~m}$ in increments of $50 \mathrm{~m}$. At each point, a series of 10 measurements of the signal level of the gateway by the tester and the tester signal by the gateway was carried out. According to the results, the average Received Signal Strength Indicator (RSSI) value was determined. Then radio signal attenuation coefficients in different conditions were calculated according to the equation 1 (Shpenst, 2015). 


$$
R S S I=T S S I+b \cdot \log 10(x),
$$

where $R S S I$ - Received Signal Strength Indicator, $\mathrm{dBm}$;

$T S S I$ - radio signal level at a distance of $1 \mathrm{~m}$ from the transmitting antenna, $\mathrm{dBm}$;

$b$ - radio signal attenuation coefficient, $\mathrm{dBm}$;

$x$ - distance between the gateway and the tester, $\mathrm{m}$.

\section{RESULTS AND DISCUSSION}

According to the measurements the coverage area of the LoRaWAN gateway in the city center was $1500 \mathrm{~m}$ and in the outskirt surrounded by coniferous forest was $1050 \mathrm{~m}$. After exceeding these distances, the signal was lost. The results of RSSI measurements in the city center and in the outskirt surrounded by coniferous forest are illustrated in Figures 4 and 5 accordingly.

The results of radio signal attenuation coefficients calculation are presented in Table 2.

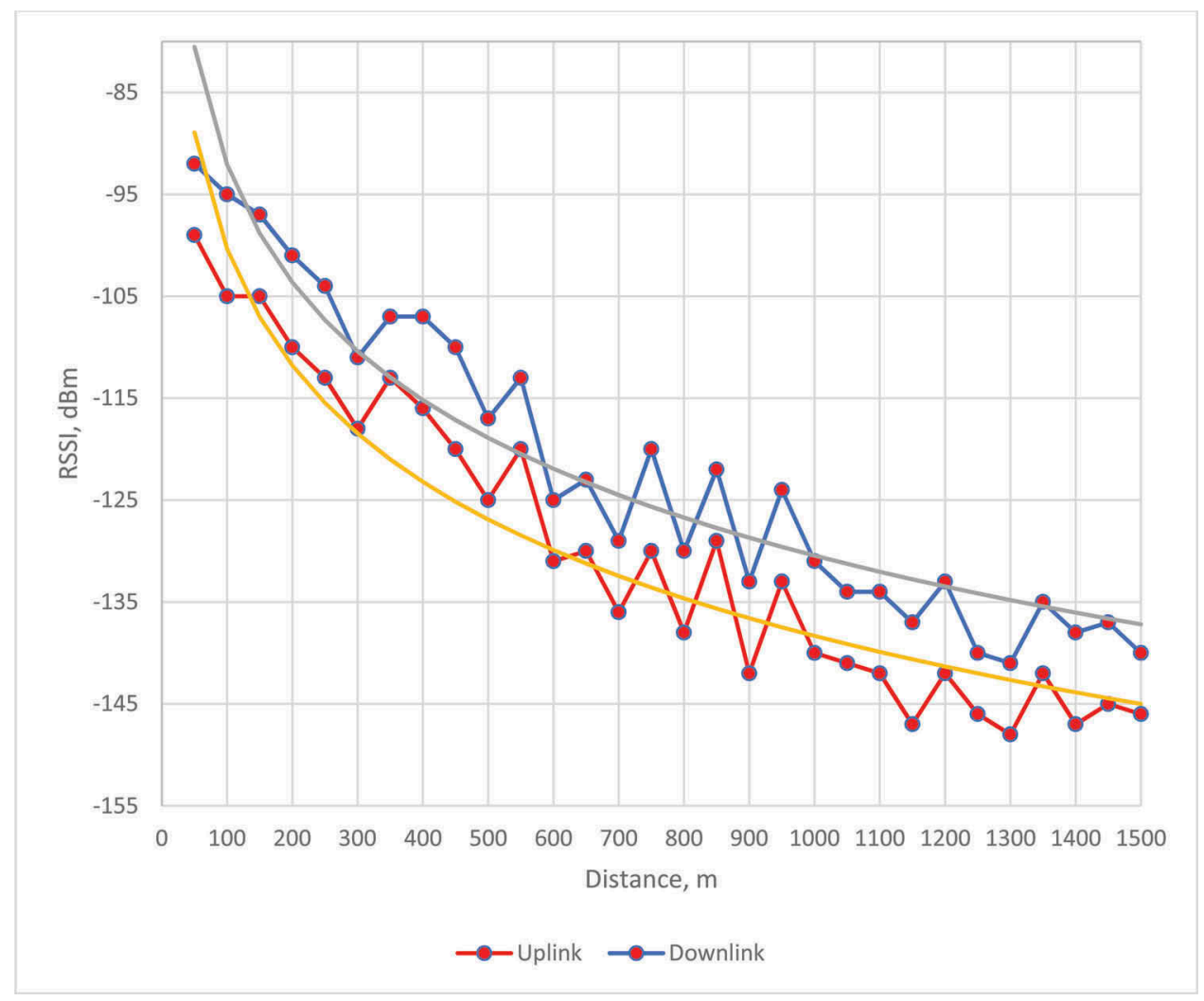

Figure 4. Dependence of LoRaWAN signal level on distance in the city center. 


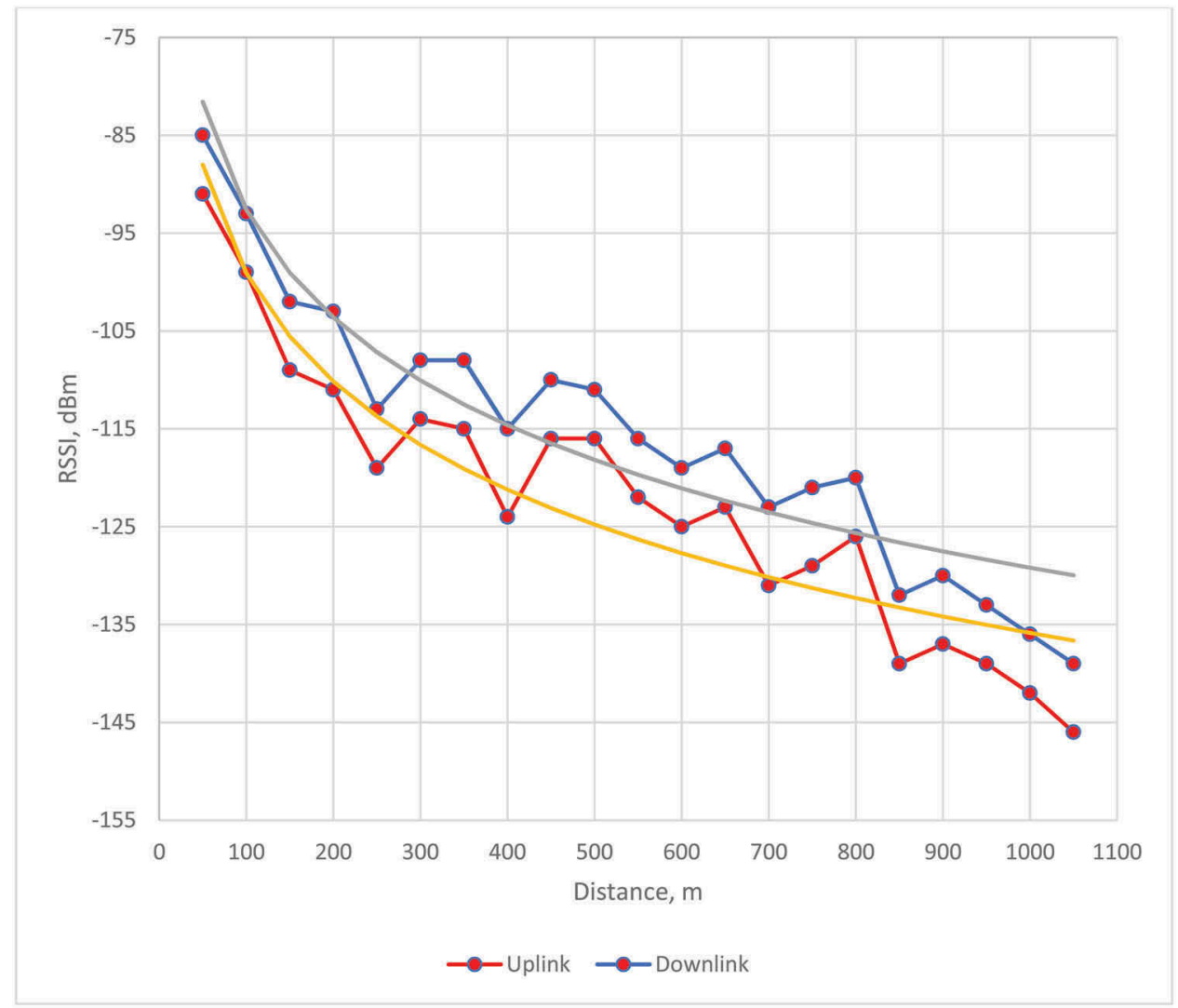

Figure 5. Dependence of LoRaWAN signal level on distance in the outskirt surrounded by coniferous forest.

Table 2. LoRaWAN signal attenuation coefficient in the conditions under the study.

\begin{tabular}{lll}
\hline Conditions & LoRaWAN signal attenuation coefficient, $\mathrm{dBm}$ \\
\hline \multirow{2}{*}{ City center } & Uplink & -24.4 \\
& Downlink & -15.4 \\
Outskirt surrounded by coniferous forest & Uplink & -25.5 \\
\cline { 2 - 3 } & Downlink & -19.4 \\
\hline
\end{tabular}

\section{CONCLUSIONS}

Rapid development of smart city services requires new network solutions to allow millions of sensors and devices to communicate with each other. In the same time, smart city applications have a number of specific requirements. The main condition of effective functioning of distributed network of devices and sensors is energy efficiency. Nowadays, smart city services based on LoRaWAN technology has the best prospects among others LPWANs due to affordability on the Russian market and good characteristics.

According to the field study results, LoRaWAN signal attenuation in conditions of coniferous forest is higher compare to the city center. It can be explained by moisture absorption in the trees.

To provide full LoRaWAN coverage for smart city services it is necessary to locate the gate ways on a distance of $1500 \mathrm{~m}$ at the city center and of $1050 \mathrm{~m}$ at the outskirt at 
maximum. It means that to cover all the area of Saint Petersburg, approximately 400 LoRaWAN gateways are needed and 84000 gateways are required for the Leningrad region.

\section{REFERENCES}

Chukin V., Kachnov Y., Preliminary results of experiments to determine the conditions of radio communication between nodes of wireless sensor networks in the frequency range of EU868 in the urban environment and forest area of the Leningrad Region, The twenty-third scientific conference on radiophysics dedicated to the 100th anniversary of the birth of N.A. Zheleztsova, Nizhny Novgorod, 05. 16.2019

Digital life of Russian megacities. Model. Dynamics. Examples//SKOLKOVO Business School Emerging Market Research Institute (IEMS) [Online]. 2016. Available at: https://iems.skolkovo.ru/downloads/ documents/SKOLKOVO_IEMS/Research_Reports/SKOLKOVO_IEMS_Re-search_2016-11-30_ru. pdf [Accessed: 16.09.2019];

Evans D., (2011), The Internet of Things: How the Next Evolution of the Internet Is Changing Everything, White paper, p.3;

Frost \& Sullivan's Global $360^{\circ}$ Research Team. Smart City Adoption Timeline. Anticipating the Global Advancement of Smart Cities; http://www.sut.ru/doci/nauka/review/20163/1-14.pdf

Indicators of smart cities NIITS 2017//NIITS [Online]. 2017. Available at: http://niitc.ru/publications/ SmartCities.pdf [Accessed: 15.09.2019];

Ishida, T. Isbister K. (2000), Digital Cities: Technologies, experiences, and future perspectives, SpringerVerlag, Berlin;

Koucheryavy A., Vladyko A., The Prospects for Research in the Field of Communications Networks on the 2017-2020 Years//Telecom IT. 2016. Vol. 4. Iss.3. pp. 1-14. Available at:

Shpenst V 2015 Optimum operating zones selection methods for multispectral overhead power lines remote sensing devices, Journal of Mining Institute 214 p.92-101

Sikora-Fernandez D., Stawasz D. The Concept Of Smart City In The Theory And Practice Of Urban Development Management//Romanian Journal of Regional Science [Online]. 2016. Available at: http://www.rrsa.ro/rjrs/V1015.Sikora.pdf .[Accessed: 25.08.2019];

Smart cities and infrastructure//United Nations Economic and Social Council [Online]. 2016. Available at: http://unctad.org/meetings/en/SessionalDocuments/ecn162016d2_en.pdf. [Accessed: 15.09.2019];

Smart Cities Market worth 1,201.69 Billion USD by 2022//Markets and Markets [Online]. Available at: https://www.marketsandmarkets.com/PressReleases/smart-cities.asp

Van der Meer, A. Van Winden, W., (2003), E-governance in Cities: A Comparison of Urban Information and Communication Technology Policies, Regional Studies, Vol. 37, No.4, pp. 407-419 